\title{
Situs ambiguus
}

INSERM

\section{Source}

INSERM. (1999). Orphanet: an online rare disease and orphan drug data base. Situs ambiguUs. ORPHA:157769

A rare, genetic, developmental defect during embryogenesis characterized by a partial mirror-image transposition of intra-thoracic and/or intra-abdominal org ans across the left-right axis of the body. Intra-organ variations and other malformations, such as ciliary motricity anomalies (e.g. Kartagener syndrome), biliary atresia and cardiac defects, are frequently associated. Left (polysplenia syndrome) or right (asplenia syndrome) isomerism are usually observed. 